\title{
Proportionate Sign Subband Adpative Filtering Algorithm for Network Echo Cancellers
}

\author{
Ji-Hye Seo, Sang Mok Jung, and Poo Gyeon Park
}

\begin{abstract}
This paper proposes a proportionate sign subband adaptive filtering (PSSAF) and an improved PSSAF (IPSSAF) algorithm for network echo cancellers that deal with impulsive interferences and sparse echo paths. Based on a sign subband adaptive filtering (SSAF) algorithm that is robust against impulsive interferences, the proposed algorithm minimizes $L_{1}$-norm of the subband a posteriori error vector subject to a weighted constraint on the filter coefficients. A positive definite weighting matrix is used for the constraint. In this paper, we adopt a diagonal proportionate matrix for the constraint to achieve fast initial convergence rate when the impulse response is sparse. The components of it is roughly proportional to the absolute value of current estimate of the filter, so the resulting algorithm is called PSSAF algorithm. To achieve fast initial convergence rate even for rather non-sparse impulse responses, an improved proportionate matrix is also used for the constraint, and the resulting algorithm is named as IPSSAF algorithm. Experimental results show that the proposed algorithms are more robust against highly correlated input signals, impulsive interferences, and double-talk than the original normalized subband adaptive filtering algorithm and the class of proportionate subband adaptive filtering algorithms.
\end{abstract}

Index Terms-Adaptive filters, proportionate, sign algorithm, subband adaptive filtering algorithm, weighted constraint, weighting matrix.

\section{INTRODUCTION}

A network echo cancellers (NEC) is an adaptive filter that is one of the best solutions to control acoustic echos of the voice communication networks. An adaptive filter estimates the echo path of the network and generates a replica of the room impulse response, and then the output of the filter is subtracted from the near-end signal to obtain clean signals. The scheme of the NEC is the same as a classical system identification, but there are several challenging problems for modern voice transmission over telephone networks. The first one is about the characteristics of the echo path. The length of the echo path for NEC is extremely long due to long delays caused by large scale network. Thus, the adaptive filter usually requires large number of taps, and 512 or 1024 taps

Manuscript received September 2, 2014; revised March 23, 2015. This research was supported by the MSIP (Ministry of Science, ICT and Future Planning), Korea, under the "IT Consilience Creative Program" (NIPA-2014-H0201-14-1001) supervised by the NIPA (National IT Industry Promotion Agency).

Ji-Hye Seo is with the Division of IT Convergence Engineering, Pohang University of Science and Technology, Pohang, Gyeongbuk, Korea (e-mail: bmclubhs@postech.ac.kr).

Sang Mok Jung and Poo Gyeon Park are with the Department of Electrical Engineering, Pohang University of Science and Technology, Pohang, Gyeongbuk, Korea (e-mail: illus2@postech.ac.kr, ppg@postech.ac.kr) are chosen as standard for the NEC. With long impulse response, however, most of the coefficients are close to zero; it means the typical network impulse response is sparse. Second, the excitation signals used for NEC are speech signals which are highly correlated and non-Gaussian process, so many adaptive filtering algorithms suffer from reduced convergence rate. In addition, the background noise can also be highly non-stationary or strong interferences. The third aspect to be considered in NEC is double-talk scenario which means that far-end and near-end speech are active simultaneously. In this case, a near-end speech acts like a large disturbance of the filter, so it may causes extremely slow convergence or divergence.

When developing adaptive filtering algorithms used for NEC, these challenging problems should be considered. An ideal algorithm not only should have fast convergence rate and good tracking performance but also achieves small steady-state estimation error even for long-length echo path. These problems should be maintained in the case of non-stationary excitation signal. Furthermore, the algorithm should be robust against variation of the microphone signal (background noise variations and double-talk situation). The conventional normalized least mean squares (NLMS) algorithm is the most widely used adaptive filtering algorithm due to its simplicity and robustness [1], [2]. However, for NEC, the NLMS algorithm suffers from reduced convergence rate due to long length of the filter. It is possible to consider the recursive least squares (RLS) algorithm and the affine projection algorithm (APA) [3] for obtaining fast convergence rate, but they are not appropriate for NEC application in practice because of their heavy computational complexity. Thus, there are requirements for adaptive filtering algorithms with fast convergence rate and low computational complexity to replace the NLMS algorithm.

The network echo path impulse response is known as sparse in nature. That is, only a small portion of the coefficients is active and other coefficients are zero or unnoticeably small values (inactive). Many adaptive filtering algorithm take advantage of sparseness the echo path and propose some methods to improve the convergence rate and robustness or lower computational complexity. The proportionate NLMS (PNLMS) [4] has been developed to achieve fast initial convergence rate. It updates coefficients of the weight vector by assigning different step sizes to different coefficients based on their magnitude of current estimates. For a large coefficient, a large value of step size is assigned in the update process, so this method allows to obtain fast initial convergence rate for sparse impulse responses relatively independent of their length for sparse impulse responses. However, the PNLMS algorithm slows down after its fast 
initial convergence, and it has slower convergence rate than NLMS when the network impulse response is not sparse enough. This problem has been addressed in several papers, and modifications to PNLMS algorithm is studied to improve the performance. The improved PNLMS (IPNLMS) [5] is proposed to avoid degradation of the performance for the non-sparse impulse response. It gives more optimal step sizes for given sparseness by using a control factor, so maintains its fast convergence rate even in the case of non-sparse impulse responses. To keep the fast convergence rate during the whold adaptation process, the $\mu$-law PNLMS (MPNLMS) is proposed in [6]. Also, the improved IPNLMS (IIPNLMS) [7] is proposed where the step sizes assigned for different coefficients are determined by two different relationships depending on whether the coefficient is considered as active or inactive. These concept of proportionate-type algorithms are extended to a normalized subband adaptive filtering (NSAF) algorithm [8] in [9]. The NSAF algorithm decomposes the input signals into multiple subbands in order to whiten the input signals in each subband, so the algorithm is suitable to achieve faster convergence rate than the NLMS algorithm. The computational complexity of the NSAF algorithm is not heavy comparing to the RLS or APA, and thus, the proportionate-type subband adaptive filtering algorithms are proper to use in NEC applications.

In addition to fast convergence rate and low complexity, a robustness can be an considerable issue for adaptive filtering algorithms. In particular, double-talk is a big challenging problem for NEC. To deal with double-talk situation, a double-talk detector (DTD) can be used typically. However, it is not desirable to rely only on DTD because the NEC has tight requirements for detecting double-talk. Thus, several adaptive filtering algorithms are developed focusing on the robustness to double-talk [10]-[14]. One of the method is using $L_{1}$-norm minimization of error because $L_{1}$-norm algorithms are especially robust against impulsive noise such as speech. The basic algorithm using $L_{1}$-norm is the normalized sign algorithm (NSA). The NSA provides robustness against impulsive noise, but the convergence rate of it becomes slower than the NLMS algorithm. Recently, a sign subband adaptive filtering (SSAF) algorithm is suggested in [15] which extends a concept of $L_{1}$-norm error minimization to the NSAF. The SSAF algorithm has good robustness comparing to NSAF algorithm, and achieves rather faster convergence rate than the NSA.

In this paper, we adopt the proportionate approach with the SSAF to obtain faster convergence for sparse network impulse responses. Based on the weighted constraint to the weight error vector, we obtained a weight update equation for the proposed algorithm mathematical derivation. Two types of proportionate matrix based on the PNLMS [4] and IPNLMS [5] are used as a weighting matrix, and the resulting algorithms are called proportionate sign subband adaptive filtering (PSSAF) algorithm and improved PSSAF (IPSSAF) algorithm, respectively. The PSSAF and IPSSAF algorithm show fast convergence rate for sparse impulse response, and they also show the robustness against highly correlated input signal, interferences, and double-talk. Especially, the IPSSAF algorithm also achieves fast convergence rate even for the non-sparse impulse response. The computational complexity of the proposed algorithms are slightly higher than the SSAF algorithm, but lower than the NSAF and the family or proportionate-type NSAF algorithms.

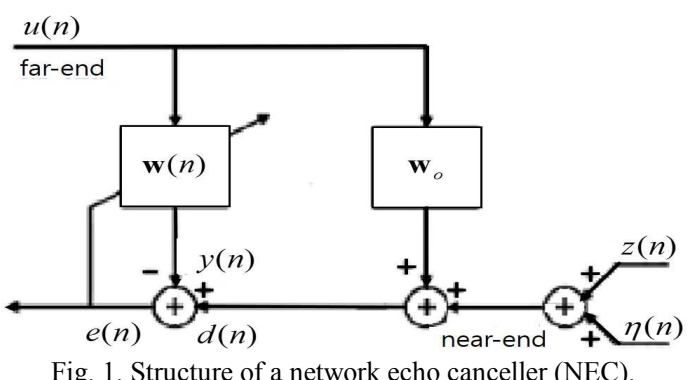

\section{BACKGROUNDS}

\section{A. Network Echo Cancellers}

Consider a NEC scheme shown in Fig. 1. In Fig. 1, $u(n)$ denotes the far-end signal, and $\eta(n)=z(n)+v(n)$ is a near-end signal where $z(n)$ and $v(n)$ are the near-end speech and background noise signal, respectively. The true echo path is denoted as $\mathbf{w}_{o}$ with length $M$, and the estimation of it can be obtained by minimizing the difference between the desired output $d(n)$ and the estimated filter output $y(n)$ such that $e(n)=d(n)-y(n)$. The desired output that contains the echo, the near-end signal can be represented as $d(n)=\mathbf{u}^{T}(n) \mathbf{w}_{o}+\eta(n)$, and the filter output, the replica of the echo, can be expressed as $y(n)=\mathbf{u}^{T}(n) \mathbf{w}(n)$, respectively, where $\mathbf{u}(n)=[u(n), u(n-1), \cdots, u(n-M+1)]^{T}$ is the far-end signal vector and $\mathbf{w}(n)=\left[w_{0}(n), w_{1}(n), \cdots, w_{M-1}(n)\right]^{T}$ is the estimation of the impulse response at time index $n$.

\section{B. Sign Subband Adaptive Filtering Algorithm}

Fig. 2 shows the conventional subband adaptive filtering structure. The signals $u(n)$ and $d(n)$ are decomposed into $u_{i}(n)$ and $d_{i}(n)$ by analysis filters $H_{i}(z) i=0,1, \cdots, N-1$. The subband input signals $u_{i}(n)$ are filtered by the adaptive filter $W(z)$ and the filter outputs $y_{i}(n), i=0,1, \cdots, N-1$ are obtained. By decimating $d_{i}(n)$ and $y_{i}(n), d_{i, D}(k)$ and $y_{i, D}(k)$ are generated, and it is easy to note that $y_{i, D}(k)=\mathbf{u}_{i}^{T}(k) \mathbf{w}(k)$, where $\mathbf{w}(k)$ is the tap weight vector of $W(z)$ and $\mathbf{u}_{i}(k)=\left[u_{i}(k N), u_{i}(k N-1), \cdots, u_{i}(k N-M+1)\right]^{T}$. We use $n$ and $k$ to index the original and decimated signal, respectively.

Define the subband output error vector $\mathbf{e}_{D}(k)$ and subband a posteriori error vector $\mathbf{e}_{p}(k)$ as

$$
\begin{gathered}
\mathbf{e}_{D}(k)=\mathbf{d}_{D}(k)-\mathbf{U}^{T}(k) \mathbf{w}(k) \\
\mathbf{e}_{p}(k)=\mathbf{d}_{D}(k)-\mathbf{U}^{T}(k) \mathbf{w}(k+1)
\end{gathered}
$$

where $\quad \mathbf{d}_{D}(k)=\left[d_{0, D}(k), d_{1, D}(k), \cdots, d_{N-1, D}(k)\right]^{T} \quad$ and $\mathbf{U}(k)=\left[\mathbf{u}_{0}(k), \mathbf{u}_{1}(k), \cdots, \mathbf{u}_{N-1}(k)\right]$. 


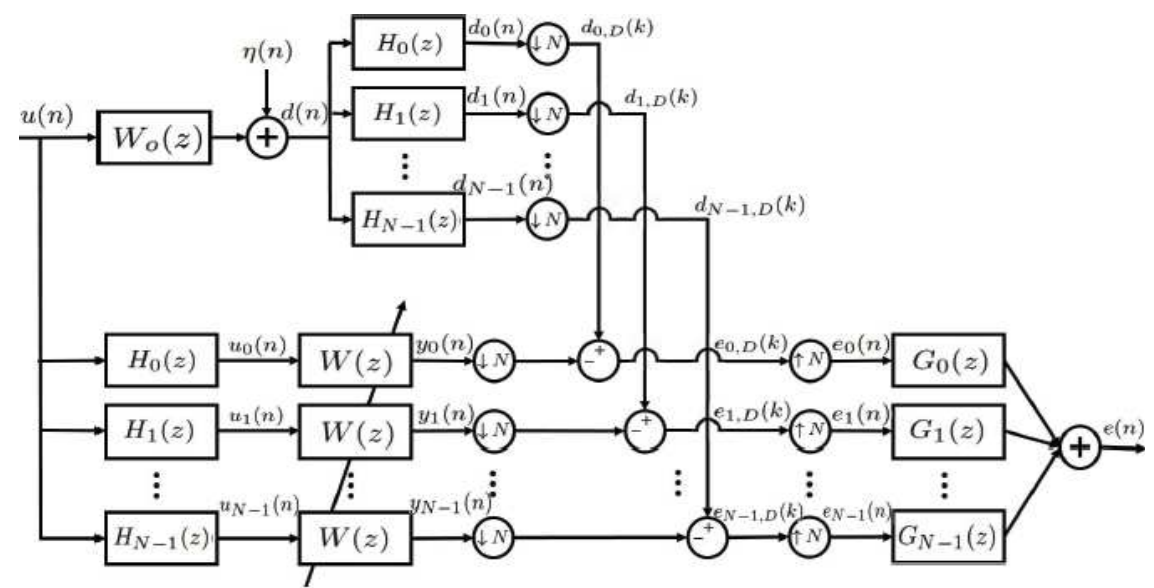

Fig. 2. Structure of NSAF algorithm.

The sign subband adaptive filtering (SSAF) algorithm [15] can be obtained by minimizing the $L_{1}$-norm of the a posteriori error vector with a constraint on the filter coefficients,

Minimizing

$$
\left\|\mathbf{d}_{D}(k)-\mathbf{U}^{T}(k) \mathbf{w}(k+1)\right\|_{1}
$$

Subject to

$$
\|\mathbf{w}(k+1)-\mathbf{w}(k)\|_{2}^{2} \leq \mu^{2}
$$

where $\mu$ is a positive step size that does not change dramatically. Using Lagrange multipliers to solve this constrained optimization problem and replacing $\mathbf{e}_{p}(k)$ to $\mathbf{e}_{D}(k)$, the weight update equation of the SSAF can be obtained as follows:

$$
\mathbf{w}(k+1)=\mathbf{w}(k)+\mu \frac{\mathbf{U}(k) \operatorname{sgn}\left(\mathbf{e}_{D}(k)\right)}{\sqrt{\operatorname{sgn}\left(\mathbf{e}_{D}^{T}(k)\right) \mathbf{U}^{T}(k) \mathbf{U}(k) \operatorname{sgn}\left(\mathbf{e}_{D}(k)\right)}}(4)
$$

Using the diagonal assumption, that is, $\mathbf{U}^{T}(k) \mathbf{U}(k)$ $=\operatorname{diag}\left[\mathbf{u}_{0}^{T}(k) \mathbf{u}_{0}(k), \mathbf{u}_{1}^{T}(k) \mathbf{u}_{1}(k), \cdots, \mathbf{u}_{N-1}^{T}(k) \mathbf{u}_{N-1}\right]$, we can write (4) as

$$
\mathbf{w}(k+1)=\mathbf{w}(k)+\mu \frac{\mathbf{U}(k) \operatorname{sgn}\left(\mathbf{e}_{D}(k)\right)}{\sqrt{\sum_{i=0}^{N-1} \mathbf{u}_{i}^{T}(k) \mathbf{u}_{i}(k)+\delta}}
$$

where $\delta$ is a regularization parameter.

\section{Proportionate Sign SUBBAnd AdAPtive FILTERING ALGORITHM}

Instead of using $L_{2}$-norm to the constraint on the filter coefficients in (3), we can use a "weighted norm with a positive definite weighting matrix $\Sigma(k)$ as

Minimizing

$$
\left\|\mathbf{d}_{D}(k)-\mathbf{U}^{T}(k) \mathbf{w}(k+1)\right\|_{1}
$$

Subject to

$$
\|\mathbf{w}(k+1)-\mathbf{w}(k)\|_{\mathbf{\Sigma}(k)}^{2} \leq \mu^{2}
$$

Using the method of Lagrange multipliers, the cost function can be obtained as

$$
\begin{gathered}
J(\mathbf{w}(k+1))=\left\|\mathbf{d}_{D}(k)-\mathbf{U}^{T}(k) \mathbf{w}(k+1)\right\|_{1} \\
+\gamma\left[\|\mathbf{w}(k+1)-\mathbf{w}(k)\|_{\Sigma(k)}^{2}-\mu^{2}\right]
\end{gathered}
$$

where $\gamma$ is a Lagrange multiplier. Setting the derivative of (7) with respect to the $\mathbf{w}(k+1)$ equal to zero, we get

$$
\mathbf{w}(k+1)=\mathbf{w}(k)+\frac{1}{2 \gamma} \Sigma^{-1}(k) \mathbf{U}(k) \operatorname{sgn}\left(\mathbf{e}_{p}(k)\right) .
$$

Since a posteriori error vector $\mathbf{e}_{p}(k)$ is not accessible before the update, we need to approximate it to a priori error vector $\mathbf{e}_{D}(k)$. Then, (8) can be replaced with

$$
\mathbf{w}(k+1)=\mathbf{w}(k)+\frac{1}{2 \gamma} \mathbf{\Sigma}^{-1}(k) \mathbf{U}(k) \operatorname{sgn}\left(\mathbf{e}_{D}(k)\right) .
$$

Substituting (9) to the constraint in (7), we obtain

$$
\frac{1}{2 \gamma}=\frac{\mu}{\sqrt{\operatorname{sgn}\left(\mathbf{e}_{D}^{T}(k)\right) \mathbf{U}^{T}(k) \Sigma^{-1}(k) \mathbf{U}(k) \operatorname{sgn}\left(\mathbf{e}_{D}(k)\right)}}
$$

Substituting (10) into (9), the update equation of the filter coefficients can be expressed as

$$
\begin{gathered}
\mathbf{w}(k+1)=\mathbf{w}(k) \\
+\mu \frac{\Sigma^{-1}(k) \mathbf{U}(k) \operatorname{sgn}\left(\mathbf{e}_{D}(k)\right)}{\sqrt{\operatorname{sgn}\left(\mathbf{e}_{D}^{T}(k)\right) \mathbf{U}^{T}(k) \Sigma^{-1}(k) \mathbf{U}(k) \operatorname{sgn}\left(\mathbf{e}_{D}(k)\right)}}
\end{gathered}
$$

We can choose the matrix $\Sigma(k)$ depending on the environment. Thus, we choose $\boldsymbol{\Sigma}(k)=\mathbf{G}^{-1}(k)$ $=\operatorname{diag}\left[1 / g_{l}(k)\right]_{l=0}^{M-1}$ as a weighting matrix, and then (11) can 
be expressed with the matrix $\mathbf{G}(k)$ as

$$
\begin{gathered}
\mathbf{w}(k+1)=\mathbf{w}(k) \\
+\mu \frac{\mathbf{G}(k) \mathbf{U}(k) \operatorname{sgn}\left(\mathbf{e}_{D}(k)\right)}{\sqrt{\operatorname{sgn}\left(\mathbf{e}_{D}^{T}(k)\right) \mathbf{U}^{T}(k) \mathbf{G}(k) \mathbf{U}(k) \operatorname{sgn}\left(\mathbf{e}_{D}(k)\right)}}
\end{gathered}
$$

The components of $\mathbf{G}(k)$ can be determined according to [4] and [5] as

$$
g_{l}(k)=\frac{\gamma_{l}(k)}{\sum_{l=0}^{M-1} \gamma_{l}(k) / M}
$$

where

$$
\gamma_{l}(k)=\max \left(\rho \max \left(q,\left|w_{0}(k)\right|, \cdots,\left|w_{M-1}(k)\right|\right),\left|w_{l}(k)\right|\right)(14
$$

or

$$
g_{l}(k)=\frac{1-\alpha}{2}+(1+\alpha) \frac{\left|w_{l}(k)\right|}{\left(2\|\mathbf{w}(k)\|_{1}+\varepsilon\right) / M} .
$$

The resulting algorithms depending on the component $g_{l}(k)$ in two ways are called proportionate sign subband adaptive filtering (PSSAF) and improved PSSAF(IPSSAF) algorithms, repectively. In (14), the parameter $\rho$ prevents $w_{l}(k)$ from stalling when it is much smaller than the largest coefficient and $q$ regularizes the updating when all coefficients are zero for the PSSAF algorithm. In (15), the parameter $\alpha$ controls the behavior of the IPSSAF algorithm. When $\alpha=-1$, the IPSSAF and SSAF are identical, and the IPSSAF behaves like the PSSAF when $\alpha$ gets closer to 1 .

\section{SimUlATION RESUlts}

\section{A. Performance Comparison}

In this section, we did several simulations to verify the performance of our proposed algorithm. The echo paths used for simulations have 512 coefficients and two typical impulse responses are depicted in Fig. 3. Two input signals are used in the simulations: the first one is obtained by filtering a white zero-mean Gaussian random sequence through the $G_{1}(z)=1 /\left(1-0.9 z^{-1}\right)$, and the second one is a speech signal. The measurement noise, $v(k)$, is added to $c(k)$ with a signal-to-noise ratio (SNR) defined by

$$
S N R=10 \log _{10}\left(\frac{E\left[c^{2}(k)\right]}{E\left[v^{2}(k)\right]}\right)
$$

where $c(k)=\mathbf{u}^{T}(k) \mathbf{w}_{o}$. The near-end signal, $z(k)$, is a strong impulsive interference with a signal-to-interference ratio (SIR) of $-30 \mathrm{~dB}$ and Bernoulli-Gaussian (BG) distribution is used for modeling the interference signal. The BG distribution is generated as the product of a Bernoulli process and a Gaussian process, i.e., $z(k)=w(k) n(k)$, where $n(k)$ is a white Gaussian random sequence with zero mean and variance $\sigma_{n}^{2}$, and $w(k)$ is a Bernoulli process with the probability mass function given as $P(w)=1-P r$ for $w=0$, and $P(w)=\operatorname{Pr}$ for $w=1$. The average power of a BG process is $\operatorname{Pr} \cdot \sigma_{n}^{2}$. Keeping the average power constant, a BG process is spikier when $\operatorname{Pr}$ is smaller. It reduces to a Gaussian process when $P r=1$. The MSD, i.e., $E\left\|\mathbf{w}_{o}-\mathbf{w}(k)\right\|^{2}$, is an indicator of the performance of the algorithms, and it is obtained by ensemble averaging over 20 independent trials. In addition, the regularization factors are set to $\delta=\sigma_{u}^{2}$ for the NSAF, PSAF, SSAF, and PSSAF algorithms, and $\delta_{I P}=(1-\alpha) / 2 \delta$ for the IPSAF and IPSSAF algorithms where $\sigma_{u}^{2}$ is a variance of input signal.

Fig. 4 shows the MSD curves for our proposed algorithms and several other algorithms under consideration for comparison. $\mathrm{AR}(1)$ process generated using $G_{1}$ was used as an input signal, and SNR was set to $30 \mathrm{~dB}$. Considering the strong interference with $P r=0.01$, the proposed PSSAF and IPSSAF algorithms show faster convergence rate than the SSAF algorithm, while the NSAF, PSAF, and IPSAF algorithm diverges because of their $L_{2}$-norm minimization of the error vector.
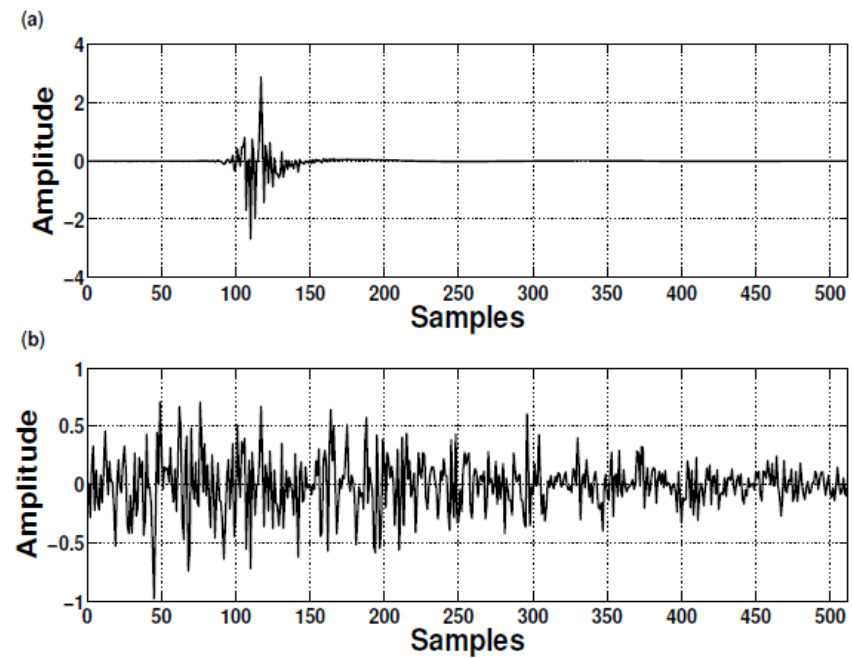

Fig. 3. Room impulse response of the echo path for NEC. (a) Sparse impulse response. (b) Dispersive impulse response.

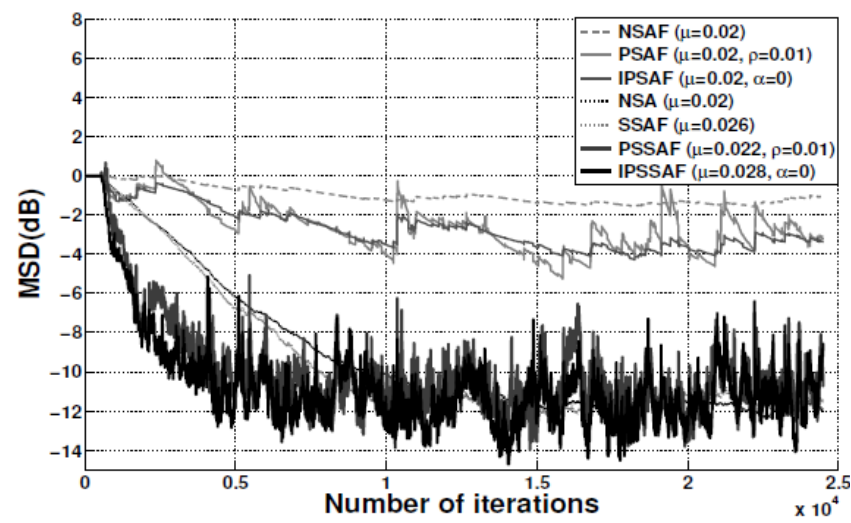

Fig. 4. MSD curves for the NSAF, PSAF, IPSAF, SSAF, and proposed PSSAF and IPSSAF algorithm with interference for sparse impulse response. Input signal is $\mathrm{AR}(1)$, and $\mathrm{SNR}=30 \mathrm{~dB}$. 


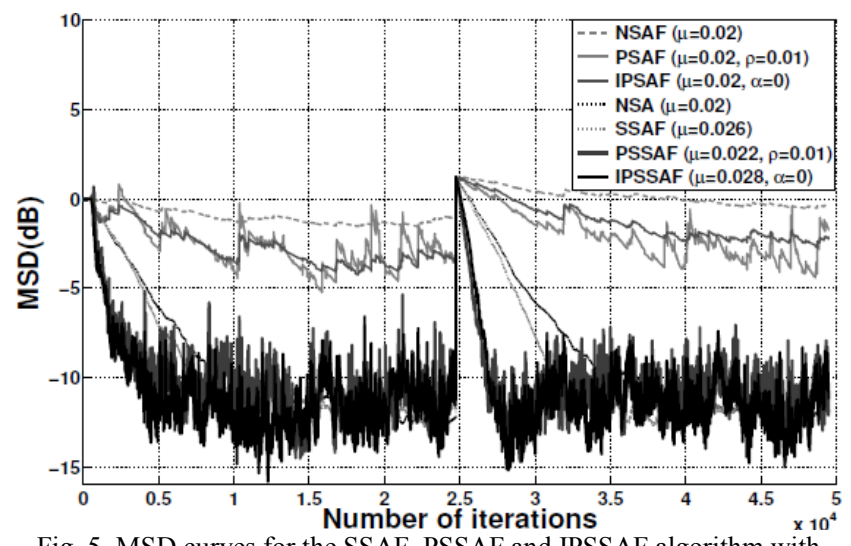

Fig. 5. MSD curves for the SSAF, PSSAF and IPSSAF algorithm with interference for sparse impulse response. Input signal is AR(1), and SNR = $30 \mathrm{~dB}$. Echo path is changed abruptly at $k=25000$.

\section{B. Tracking}

Another possible scenario in NEC is the change of the echo path. The result for this scenario is depicted in Fig. 6, where the sparse echo path (Fig. 3(a)) was abruptly changed at $k=25000$ to the dispersive impulse response (Fig. 3(b)). Environment for this simulation is the same as in Fig. 4, and the step sizes for the SSAF, PSSAF, and IPSSAF were set to make them achieve the same steady-state MSD. In Fig. 5, the IPSSAF algorithm achieves the fastest tracking performance among the comparing algorithms, and the PSSAF algorithm achieves better tracking performance than the SSAF algorithm, where the other algorithms track the changed echo path slowly.

\section{Parameters}

To verify the effects of the proportionate matrix for sparse and dispersive echo paths, we did additional simulations by varying parameters used for proportionate matrix. Fig. 6, Fig. 7 depict the results of the simulation for the PSSAF and IPSSAF algorithms, respectively. AR(1) input and BG interference was used in the simulations for impulse responses shown in Fig. 3, and the step size was set to $\mu=0.01$.

In Fig. 6(a), the PSSAF algorithm with small $\rho(\rho=0.01)$ leads to fast convergence rate for sparse impulse response because more proportionality for the filter PSSAF algorithm with larger value of $\rho$ achieves better performance for dispersive impulse response because larger $\rho$ makes little proportionality, and the algorithm updates the filter coefficients with a similar rate. We verified that the value of $\rho$ considerably affects the performance of the PSSAF algorithm depending on the sparseness of the network impulse responses. We also did experiment about the effect of $\alpha$ for the IPSSAF algorithm in Fig. 7. In Fig. 7(a), as the value of $\alpha$ get closer to -1 , the convergence rate of the IPSSAF algorithm get slower for sparse impulse response. The convergence behavior of the IPSSAF for dispersive impulse response is shown in Fig. 7(b), and we can notice that the algorithm shows similar initial convergence rate regardless of the value of $\alpha$. As the value of $\alpha$ increases, the convergence rate of the IPSSAF algorithm get slower after initial convergence, but the steady-state estimation error of the algorithm with different $\alpha$ is not much different for dispersive impulse response. The effect of $\alpha$ is less sensitive than the effect $\rho$ of the PSSAF algorithm, and it is good to choose $\alpha=0$ or $\alpha=$
-0.5 to make the IPSSAF algorithm perform robust to sparseness of the network impulse responses.
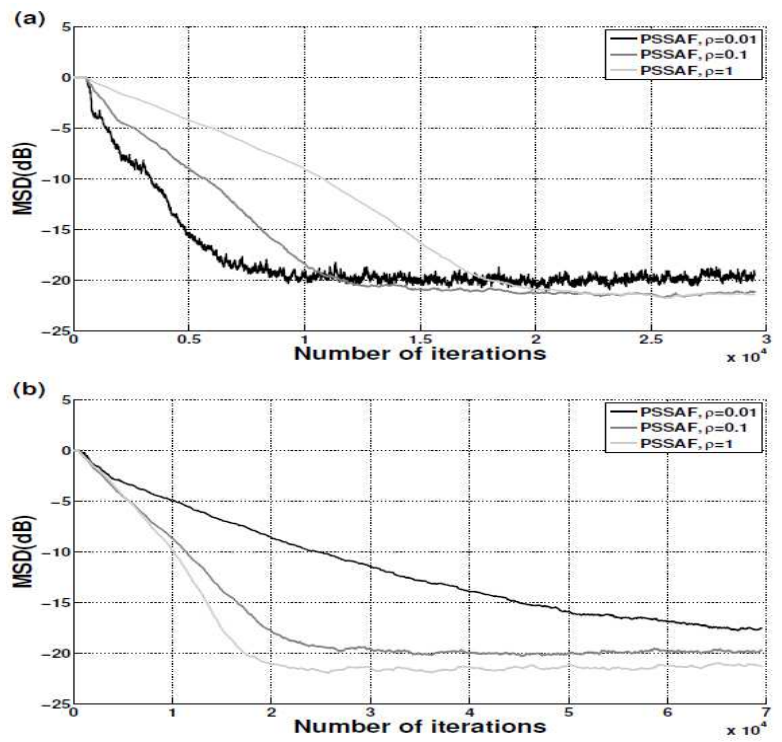

Fig. 6. MSD curves for the PSSAF algorithm for different $\rho$ for $\mu=0.01$. The simulation environment is the same as in Fig. 5. Impulse responses in Fig. 3 were used as the (a) sparse and (b) dispersive impulse responses, respectively.
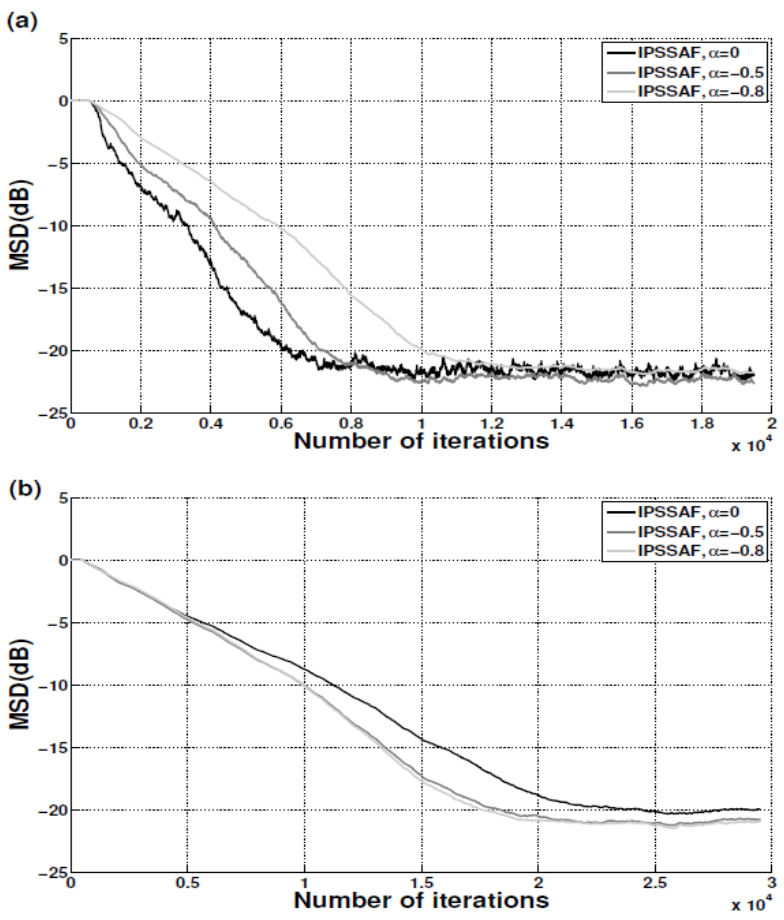

Fig. 7. MSD curves for the IPSSAF algorithm for different $\alpha$ for $\mu=0.01$. The simulation environment is the same as in Fig. 5. Impulse responses in Fig. 3 were used as the (a) sparse and (b) dispersive impulse responses, respectively.

\section{Double-Talk Scenario}

In this subsection, we show the performance of the proposed and competing algorithms in the double-talk situation. Experimental environment is the same as Fig. 4 except excitation signal, and impulse response in Fig. 3(a) is used as the network echo path. Speech signals used for double-talk scenario are depicted in Fig. 8; far-end and near-end speech, respectively. Double-talk happen in the period with sample $[2.0,4.0] \times 10^{4}$. As shown in Fig. 9 and Fig. 
10, the NSAF, PSAF, and IPSAF show non-robust performance against double-talk disturbance without and with interferences. In contrast, the NSA, SSAF, PSSAF and IPSSAF algorithm, a family of sign algorithms, show somewhat robust performance against both impulsive interferences and double-talk disturbance. Among these family of sign algorithms, the proposed PSSAF and IPSSAF achieves faster convergence rate and smaller steady-state estimation error than the NSA and SSAF algorithm. Without any double-talk detector, the proposed PSSAF and IPSSAF show better robustness to double-talk.
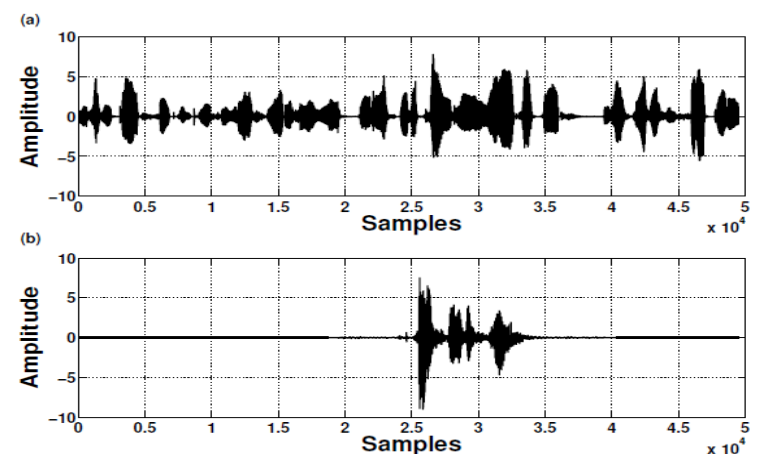

Fig. 8. Speech signals used in the double-talk scenario. (a) Far-end speech (b) Near-end speech.

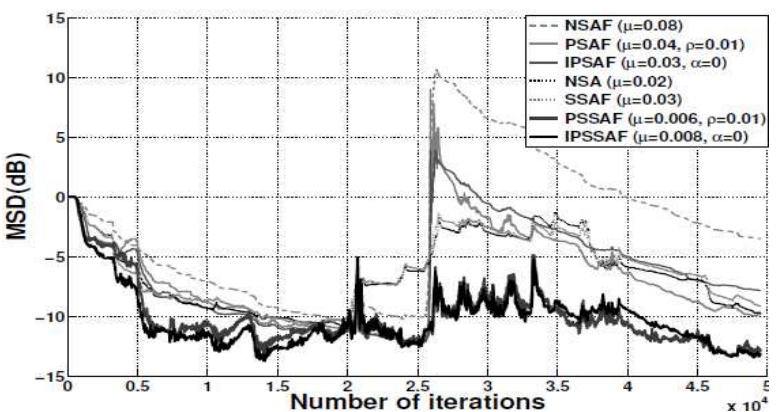

Fig. 9. MSD curves for the NSAF, PSAF, IPSAF, NSA, SSAF, and proposed PSSAF and IPSSAF algorithm for double-talk scenario without impulsive interferences.

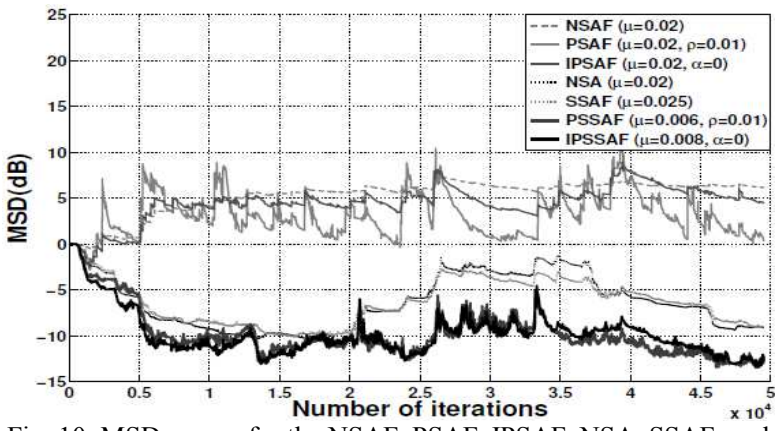

Fig. 10. MSD curves for the NSAF, PSAF, IPSAF, NSA, SSAF, and proposed PSSAF and IPSSAF algorithm for double-talk scenario with impulsive interferences.

\section{CONCLUSION}

In this paper, we proposed proportionate-type sign subband adaptive filtering algorithms for network echo cancellation. The proposed algorithm minimizes $L_{1}$-norm of the subband $a$ posteriori error vector subject to a weighted constraint. Applying proper positive-definite weighting matrix to the weighted constraint, we obtained proportionate sing subband adaptive filtering (PSSAF) algorithm and improved PSSAF
(IPSSAF) algorithm. The resulting PSSAF and IPSSAF algorithm showed faster convergence rate than the conventional $L_{1}$ algorithm for sparse network impulse response. Moreover, they achieved more robust performance against impulsive interferences and double-talk than the existing algorithms.

\section{ACKNOWLEDGMENT}

This research was supported by the MSIP (Ministry of Science, ICT and Future Planning), Korea, under the "IT Consilience Creative Program" (NIPA-2014-H0201-14-1001) supervised by the NIPA (National IT Industry Promotion Agency).

\section{REFERENCES}

[1] S. Haykin, Adaptive Filter Theory, 1st ed. Upper Saddle River, NJ: Prentice-Hall, 2001.

[2] A. H. Sayed, Fundamentals of Adaptive Filtering, New York: Wiley, 2003.

[3] K. Ozeki and T. Umeda, "An adaptive filtering algorithm using an orthogonal projection to an affine subspace and its properties," Electronics and Communications in Japan, vol. 67-A, no. 5, pp. 19-27, 1984.

[4] D. L. Duttweiler, "Proportionate normalized least-mean-squares adaptation in echo cancelers," IEEE Trans. on Speech and Audio Processing, vol. 8, no. 5, pp. 508-518, September 2000.

[5] J. Benesty and S. L. Gay, "An improved PNLMS algorithm," in Proc. IEEE International Conference on Acoustics, Speech, and Signal Process, vol. 2, pp. 1881-1884, May 2002.

[6] H. Deng and M. Doroslovacki, "Proportionate adaptive algorithms for network echo cancellation," IEEE Trans. on Signal Processing, vol. 54, pp. 1794-1803, May 2006.

[7] P. A. Naylor, J. Cui, and M. Brookes, "Adaptive algorithms for sparse echo cancellation,” Signal Processing, vol. 86, pp. 11825-1192, 2006.

[8] K. A. Lee, W. S. Gan, and S. M. Kuo, Subband Adaptive Filtering: Theory and Implementation, Wiley, 2009.

[9] M. S. E. Abadi, "Proportionate normalized subband adaptive filter algorithms for sparse system identification," Signal Processing, vol. 87, no. 7, pp. 1467-1474, July 2009.

[10] J. Benesty, H. Rey, L. R. Vega, and S. Tressens, "A nonparametric VSS NLMS algorithm," IEEE Signal Processing Letters, vol. 13, no. 10, pp. 581-584, October 2006.

[11] T. Gansler, S. L. Gay, M. M. Sondhi, and J. Benesty, "Double-talk robust fast converging algorithms for network echo cancellation," IEEE Trans. on Speech and Audio Processing, vol. 8, no. 6, pp. 656-663, November 2000.

[12] T. Waterschoot, G. Rombouts, P. Verhoeve, and M. Moonen, "Double-talk-robust prediction error identification algorithms for acoustic echo cancellation," IEEE Trans. on Signal Processing, vol. 55, no. 3, pp. 846-858, March 2007.

[13] C. Paleologu, J. Benesty, and S. Ciochina, "A variable step-size affine projection algorithm designed for acoustic echo cancellation," IEEE Trans. on Audio, Speech, and Language Processing, vol. 16, no. 8, pp. 1466-1478, November 2008.

[14] Z. Yang et al., "Proportionate affine projection sign algorithms for network echo cancellation," IEEE Trans. on Audio, Speech, and Language Processing, vol. 19, no. 8, pp. 2273-2284, November 2011.

[15] J. Ni and F. Li, "Variable regularisation parameter sign subband adaptive filter," Electronics Letters, vol. 46, no. 24, pp. 1605-1607, November 2010.

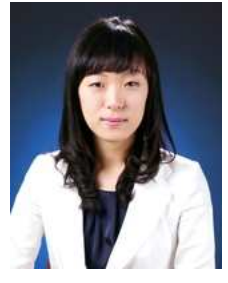

Ji-Hye Seo received the B.Sc. degree in electrical engineering from Pohang University of Science and Technology (POSTECH), Pohang, Korea, in 2010, and the M.Sc. degree from the Division of IT Convergence Engineering from POSTECH. Currently, she is a Ph.D. candidate in the Division of IT Convergence Engineering, POSTECH, in 2012.

Her main research interests include signal processing. processing, adaptive filtering algorithm, and image 


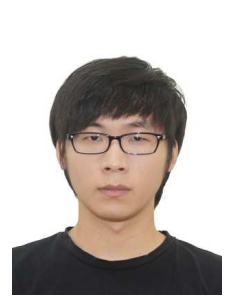

Sang Mok Jung received the B.Sc. and M.Sc. degrees in electrical engineering from Pohang University of Science and Technology (POSTECH), Pohang, Korea, in 2010 and 2012, respectively. Currently, he is a $\mathrm{Ph} . \mathrm{D}$. degree candidate in the Division of Electrical Engineering, POSTECH.

His main research interests include signal

processing, adaptive filtering algorithm, image
inspections, network programming, and their implementations.

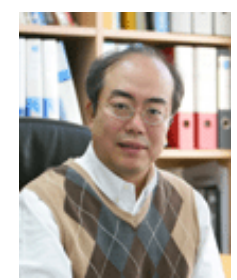

Poo Gyeon Park received the B.S. degree and M.S degree in control and instrumentation engineering from Seoul National University, Korea, in 1988 and 1990, respectively, and the Ph.D. degree in electrical engineering from Stanford University, Stanford, CA, in 1995 .

Since 1996, he has been affiliated with the Division of Electrical Engineering, Pohang University of Science and Technology, where he is currently a professor. His current research interests include robust, LPV, and network-related control theories, delayed systems, fuzzy systems, signal processing, and wireless communications for personal area network (PAN). 